\title{
LFA-1 Binding to Ligand Induces Talin-Mediated Reorganization of the Actin Cytoskeleton in Cytotoxic T Cells
}

\author{
Emily M. Mace ${ }^{\mathrm{a}}$, Matthew A. MacLeod ${ }^{\mathrm{a}}$, Muhammad Reza Marwali ${ }^{\mathrm{a}, \mathrm{b}}$, Lisa Dreolini ${ }^{\mathrm{a}, \mathrm{c}}$ and \\ Fumio Takei ${ }^{\mathrm{a}, \mathrm{d}}$
}

\author{
${ }^{a}$ Terry Fox Laboratory, British Columbia Cancer Agency, Vancouver, BC, V5Z 1L3, Canada \\ ${ }^{b}$ Current address: Department of Internal Medicine, University of Arkansas for Medical Sciences, 4301 West Markham, \\ Slot 634, Little Rock, AR 72205, USA \\ ${ }^{c}$ Current address: Genome Science Centre, BC Cancer Research Centre, 675 West 10th Avenue, Vancouver, BC, V5Z \\ IL3, Canada \\ ${ }^{d}$ Department of Pathology and Laboratory Medicine, Faculty of Medicine, University of British Columbia, Vancouver, \\ BC, V6T 2B5, Canada
}

\begin{abstract}
LFA-1 mediates binding of cytotoxic T lymphocytes (CTLs) to target cells. However, the role of LFA-1 in CTLs beyond its cell adhesion function has been unclear. We have investigated the role of LFA-1 in reorganization of the actin cytoskeleton, an essential step in cell-mediated cytotoxicity. Binding of LFA-1 to ICAM-1, but not anti-LFA-1 cross-linking, induces the accumulation of talin, WASP and actin in an antigen independent manner. Antibody crosslinking of LFA-1 on CTLs induces its capping, but talin, actin and WASP remain evenly distributed, indicating that they do not constitutively associate with LFA-1 in CTLs. These results show that ligand binding, but not simple clustering of LFA-1, induces its association with talin and provides stimulatory signals in CTLs leading to the reorganization of the actin cytoskeleton independently of TCR-mediated signaling.
\end{abstract}

Keywords: LFA-1, T lymphocytes, adhesion molecules, ICAM-1, cytotoxic T cell, actin, talin.

\section{INTRODUCTION}

LFA-1 plays a critical role in cell mediated cytotoxicity, by mediating binding of effector cells to target cells [1]. However, whether LFA-1 plays an additional role in cellmediated killing of target cells by transducing "outside-in signals" has been unclear. One of the difficulties in studying LFA-1-mediated signalling events is that LFA-1 on resting T cells has very low avidity for ligand and does not effectively mediate binding of resting $\mathrm{T}$ cells to APC. When resting $\mathrm{T}$ cells interact with APCs, LFA-1 on T cells is thought to be activated by signals generated by the TCR recognizing agonist MHC/peptide complex on APCs [2]. The activated LFA1 on $\mathrm{T}$ cells then binds to ICAM- 1 on APC to stabilize T cell-APC interaction. Thus, intracellular events mediated by LFA-1 are difficult to differentially recognize over the strong TCR-mediated signalling events. To circumvent this problem, antibody-mediated cross-linking of LFA-1 is often used to study LFA-1-mediated stimulatory signals. However, our current study shows that ligand-binding and antibodymediated clustering of LFA-1 have different effects, and cross-linking with antibody may not be physiologically relevant.

*Address correspondence to this author at the Terry Fox Laboratory, BC Cancer Agency, 675 W. 10th Ave., Vancouver, B.C., V5Z 1L3, Canada; Tel: (604) 675-8131; Fax: (604) 877-0712; E-mail: ftakei@ bccrc.ca
Porter et al. have shown that binding of primary human $\mathrm{T}$ cells to immobilized ICAM-1 induced by treatment with EGTA and $\mathrm{Mg}^{++}$to activate LFA-1 results in reorganization of the actin cytoskeleton [3]. In our previous studies, we showed that LFA-1 molecules on CTLs generated in vitro from HY-specific TCR-transgenic mouse T cells are in a high-avidity conformation and readily mediate cell adhesion to ICAM-1. Furthermore, binding of CTLs to the murine fibroblast $\mathrm{L}$ cell line expressing ICAM-1 but not agonist peptide/MHC complex leads to antigen-independent formation of the immunological synapse, in which LFA-1, CD3 and GM1 show a ring-like distribution at the cell contact site [4]. The actin cytoskeleton also accumulates at the immunological synapse of CTLs in an antigen-independent manner. Somersalo et al. have shown that binding of CTLs to a planar lipid bilayer containing ICAM-1 leads to formation of an antigen-independent ring junction consisting of LFA-1 and the actin-binding protein talin [5]. These results suggest that LFA-1-mediated binding of CTLs to ICAM-1 leads to the reorganization of the actin cytoskeleton and formation of antigen-independent immunological synapse.

Talin is a major cytoskeletal protein that has recently been proposed to act as the final common step in integrin activation [6]. It is composed of a $\sim 50 \mathrm{kDa}$ head and a $\sim 200$ $\mathrm{kDa}$ tail domain. The talin head contains a predicted FERM (band four-point-one, ezrin, radixin and moesin) domain that mediates interactions with the cytoplasmic tails of $\beta 1$ [7] $\beta 2$ 
[8] and $\beta 3[6,9]$ integrins. Overexpression of recombinant talin fragments containing this FERM domain activates $\beta 2$ [8] and $\beta 3$ [7, 9-11] integrins, possibly by disrupting the interactions between the $\alpha$ and $\beta$ cytoplasmic tails. RNAi experiments indicate that talin is required for activation of $\beta 1$ and $\beta 3$ integrins [6]. Thus, talin is thought to play a critical role in inside-out activation of LFA-1. Whether talin is involved in LFA-1-mediated outside-in signaling is currently unknown.

Wiskott Aldrich Syndrome Protein (WASP) is a leukocyte-specific member of the WASP family of proteins [12]. Upon T cell activation, WASP interacts with the actin nucleating complex Arp $2 / 3$ and promotes actin polymerization [12]. WASP is recruited to the IS formed in T cells as they interact with APC expressing agonist peptide/MHC complex, and WASP-evoked actin polymerization is thought to promote synapse formation and $\mathrm{T}$ cell activation [13], although WASP-deficient $\mathrm{T}$ cells are able to form the immunological synapse [14]. WASP-deficient NK cells have defective cytotoxicity and actin accumulation at the IS on NK cells [15].

Interaction between the LFA-1 and the actin cytoskeleton is complex. LFA-1 in resting cells is thought to be kept inactive through anchoring interactions with the actin cytoskeleton that prevent it from clustering and forming multivalent interactions with ligand [2]. After TCR engagement, intracellular signals are generated within $\mathrm{T}$ cells that are believed to cause the transient solubilization of the cortical cytoskeleton, which liberates LFA-1 and allows its lateral movement in the cell membrane, leading to multivalent interactions with ICAM-1 at the site of conjugate formation. Single molecule tracking experiments with Epstein-Barr Virus (EBV) transformed B cells showed that almost all LFA-1 molecules in resting cells were found to be immobile on the cell surface while treatment of these cells with the phorbol ester phorbol 12-myristate 13-acetate (PMA), which experimentally activates LFA-1, increased the mobility of the LFA-1 molecules by a factor of ten [16]. Additionally, treatment with low concentrations of cytochalasin D, which disrupts actin filaments and inhibits actin polymerization, enhanced cell adhesion to ICAM-1 coated surfaces. On the other hand, high concentration of cytochalasin D inhibits cell adhesion to ICAM-1. It was thought that cytochalasin induces the release of LFA-1 from actin constraints, thus allowing it to cluster whereas higher concentration of cytochalasin treatment inhibits re-association of clustered LFA-1 with actin filaments, which is needed for firm cell adhesion.

In this study, we investigated the interaction between LFA-1, talin and actin in CTLs. Our results show that ICAM-1 binding to LFA-1, but not LFA-1 clustering, is critical for the reorganization of the actin cytoskeleton. We have shown that some signaling events following LFA-1 ligation, such as those that result in actin, talin and WASP accumulation are dependent upon ICAM-1 ligation. Others, such as Pyk2 phosphorylation, can be induced by clustering of LFA-1. These results, taken together with the accumulation of actin following binding of LFA-1 to ICAM, suggest that outside-in signals are transduced following binding of LFA-1 to ICAM-1. Thus we have shown early events in a previously undefined pathway of outside-in signaling.

\section{MATERIALS AND METHODOLOGY}

\section{Mice, Antibodies and Reagents}

C57BL/6 mice were bred in our animal colony. HYspecific TCR transgenic Rag $2^{-/-}$C57BL/10 mice were obtained from Taconic Farms (Tarrytown, NY) and bred in the Joint Animal Facility of the BC Cancer Research Centre. Mice were maintained in compliance with the regulations of the Canadian Council on Animal Care, and the use of mice for this study was approved by the animal care committee of University of British Columbia. Anti-talin mAb (clone 8d4) was from Sigma (St. Louis, MO). Rat anti-murine CD18 hybridoma (TIB213) was from the American Type Culture Collection (ATCC, Rockville, MD). The YN1/1 mAb specific for murine ICAM-1 has been described [17]. Mouse anti-WASP mAb were from Santa Cruz Biotechnology (Santa Cruz, CA). Murine recombinant soluble ICAM-1 (sICAM-1) [18] was from StemCell Technologies (Vancouver BC, Canada). Cytochalasin D, bovine serum albumin (BSA) fragment V were from Sigma-Aldrich (St. Louis, MO). Calcein-AM, Alexa Fluor 488-conjugated goat anti-rat Ig secondary antibodies, Alexa Fluor 488-conjugated goat anti mouse and rhodamine-conjugated phalloidin, were from Molecular Probes (Eugene, OR).

\section{Cells}

CTLs were generated from HY-specific TCR transgenic Rag2-deficient female B10 mice by stimulating with B6 dendritic cells pulsed with HY peptide in the presence of IL2 as described [4]. The CTLs thus generated showed strong cytotoxicity against target cells expressing $\mathrm{H}-2 \mathrm{~K}^{\mathrm{b}}$ pulsed with the HY peptide. Splenic T cells were isolated by the murine $\mathrm{T}$ cell enrichment kit Spin Sep ${ }^{\mathrm{TM}}$ (StemCell Technologies, Vancouver, BC, Canada) as described [19]. The murine fibroblast $\mathrm{L}$ cells and EL4 murine leukemia line were from the ATCC (Rockville, MD). L cells transfected with ICAM-1 have been described [4].

\section{Binding of Cells to Beads Coated with ICAM-1 or Anti- LFA-1 mAb}

Polystyrene beads (polystyrene 10 micron microspheres, Polysciences Inc., Warrington PA) were mixed at a 1:1 ratio with $40 \mu \mathrm{g} / \mathrm{ml}$ solutions of anti-LFA-1 mAb (TIB213) or recombinant soluble ICAM-1 in Adhesion Buffer $(20 \mathrm{mM}$ Tris- $\mathrm{HCl}, 150 \mathrm{mM} \mathrm{NaCl}, \mathrm{pH}$ 8.2) for $1 \mathrm{~h}$ at room temperature with occasional agitation to prevent settling. Coated beads were blocked with Adhesion Buffer containing 10 $\mathrm{mg} / \mathrm{ml} \mathrm{BSA}$ for $1 \mathrm{~h}$ at room temperature, followed by three washes with RPMI 1640 10\% FCS. CTLs were harvested, centrifuged and resuspended in RPMI $10 \%$ FCS, mixed with coated beads at a 1:2 ratio in a microfuge tube and incubated at $37^{\circ} \mathrm{C}$ for 5 minutes. The cell-bead mixture was gently resuspended and plated onto a Poly-L-lysine coated coverslip, followed by incubation at $37^{\circ} \mathrm{C}$ for various time-points.

\section{Confocal Microscopy}

Induction of LFA-1 capping by antibody-mediated crosslinking and the preparation of CTLs interacting with ICAM1-transfected L cells for confocal microscopy has been described [4]. For confocal analysis of CTLs binding beads, the samples prepared above were fixed with $4 \%$ paraformalde- 
hyde for 15 minutes, blocked with PBS with 1\% BSA for 30 minutes and permeabilized with Hank's Saponin solution (HBSS containing 2\% FCS, 5 mM EDTA, 0.5\% Saponin) for 5 minutes at room temperature. Actin was detected by incubating cells fixed on coverslips with a 1:40 solution of rhodamine phalloidin in Hank's Saponin solution for 20 minutes at room temperature. WASP and Pyk-2 were stained by incubating coverslips with a 1:40 solution of the corresponding primary antibody in Hank's Saponin solution for 1 $\mathrm{h}$ at room temperature followed by incubation with $10 \mu \mathrm{g} / \mathrm{ml}$ Alex Fluor 488- or Alexa Fluor 568-conjugated goat antimouse IgG antibody for WASP, or Alex Fluor 488conjugated rabbit anti-goat IgG antibody for Pyk-2. Coverslips were washed 3 times with Hank's Saponin solution and twice with HBSS and mounted on slides using Vectashield Hard Set Antifade Mounting Medium (Vector Laboratories, Inc., Burlingame, CA). CTL-bead conjugates were analyzed using a BioRad Radiance 2000 Multiphoton microscope (Hercules, CA) with a 60x objective lens. The lasers used were $\mathrm{Kr}$ and Mai Tai Ti Sapphire. Alexa Fluor 488 was excited at $488 \mathrm{~nm}$ and the emission filter was HQ 515/30. Alexa Fluor 568 and rhodamine were excited at $568 \mathrm{~nm}$ and the emission filter was HQ 600/50. Stacks were collected using $0.15 \mu \mathrm{m} \mathrm{Z}$ steps and reconstructed using Volocity software (Improvision, Lexington, MA). The fluorescence intensity and area of actin, talin and WASP staining were measured using Volocity software on conjugates collected under identical confocal conditions. The "Integrated Intensity" was determined to be the total fluorescence within an encircled selection divided by the area.

\section{Pyk2 Phosphorylation}

Polystyrene $60 \times 15 \mathrm{~mm}$ Petri dishes (BD Falcon) were coated with $10 \mu \mathrm{g} / \mathrm{ml}$ ICAM-1 or anti-LFA-1 antibody (TIB213) in Adhesion Buffer (20 mM Tris- $\mathrm{HCl}, 150 \mathrm{mM}$ $\mathrm{NaCl} \mathrm{pH}$ 8.2) overnight at $4^{\circ} \mathrm{C}$. ICAM-1 and TIB213 coated plates were blocked with Adhesion Buffer containing $1 \%$ BSA for 1 hour at room temperature. Coated plates were washed three times with PBS before use. CTLs were harvested, centrifuged and resuspended in $37^{\circ} \mathrm{C}$ RPMI 1640 $10 \%$ FBS at 10 million cells $/ \mathrm{ml}$. One millilitre (10 million cells) of cell suspension was added to each coated plate. Plates were incubated at $37^{\circ} \mathrm{C}$ for various time-points, washed twice with $1 \mathrm{ml}$ of pre-warmed RPMI $164010 \%$ FCS, then cells were lysed with $1 \mathrm{ml}$ of ice cold lysis buffer (10 mM Tris- $\mathrm{HCl}, \mathrm{pH} 8.0,150 \mathrm{mM} \mathrm{KCl}, 2$ mM EDTA $\mathrm{pH}$ 8.0, $1 \%$ Triton X-100, 0.5\% BSA, $1 \mathrm{mM} \mathrm{Na}_{3} \mathrm{VO}_{4}, 0.2 \mathrm{mM}$ $\mathrm{NaMoO}_{4}, 1 \mathrm{mM}$ PMSF, $5 \mu \mathrm{g} / \mathrm{ml}$ aprotinin, $10 \mu \mathrm{g} / \mathrm{ml} \mathrm{leu-}$ peptin and $10 \mu \mathrm{g} / \mathrm{ml}$ pepstatin). For time zero time-points, 10 million CTLs were lysed in a microfuge tube. Cells were sheared with two passages through a \# $26 \mathrm{G}$ needle followed by incubation on ice for 10 minutes. After centrifugation at $13,000 \mathrm{rpm}$ with for 20 minutes at $4^{\circ} \mathrm{C}$, supernatants were taken as cell lysates. Immunoprecipitations were performed by incubating lysates with $1 \mu \mathrm{g}$ anti-Pyk- 2 for 1 hour at $4^{\circ} \mathrm{C}$ with constant agitation. Protein $\mathrm{G}$ beads (Amersham Biosciences, Piscataway NJ) were added and lysates were agitated for an additional hour at $4^{\circ} \mathrm{C}$. Protein $\mathrm{G}$ beads were washed three times with Wash Buffer (lysis buffer without BSA) and the immunoprecipitated proteins eluted with
SDS-PAGE sample buffer and boiling for 5 minutes. Samples for each time-point $\left(10^{7}\right.$ cell equivalent $)$ were divided in two equal portions, each separated by SDS-PAGE, blotted to polyvinylidene fluoride membranes (Pall, Pensacola, FL) and detected by anti-Pyk2 or anti-phosphotyrosine (4G10) antibody with horseradish peroxidase-conjugated secondary antibody and an ECL system (Amersham Biosciences, Piscataway, NJ) according to the manufacturer's protocol. Quantitation of western blots was performed using ImageJ software (National Institutes of Health).

\section{Cell Adhesion Assay}

LFA-1-mediated cell adhesion to immobilized soluble ICAM-1 was assayed as described [18]. For the actin cytoskeleton disruption, cytochalasin D (at indicated concentration) was added and cells were incubated for 15 minutes at $37^{\circ} \mathrm{C}$. Cells were washed once and incubated with $50 \mathrm{ng} / \mathrm{ml}$ PMA for 30 minutes at $37^{\circ} \mathrm{C}$. To determine the morphology of CTLs after washing, images were taken using bright field microscopy (Leica, Richmond Hill, Canada) with 40x objective lens equipped with CCD camera (Qimaging, Burnaby, Canada).

\section{Statistics}

Student's two-tailed t-test was used for comparison of sets of matched samples. Grouped samples were analyzed using one-way ANOVA analysis.

\section{RESULTS}

\section{Actin and WASP Accumulates in the Immunological Synapse of CTLs in an Antigen-Independent Manner}

We have previously reported that LFA-1-mediated binding of CTLs to target cells induces an antigen-independent formation of the immunological synapse consisting of a ringlike co-distribution of LFA-1 and CD3 [4]. Using this system, we examined the distribution of cytoskeletal proteins in CTLs as they interact with ICAM-1-transfected L cells, which do not express agonist peptide/MHC. The conjugates were incubated for 5 and 20 minutes and were fixed, permeabilized and stained with phalloidin for F-actin (red) and anti-LFA-1 antibody (green). When CTLs bound to L cells expressing ICAM-1, F-actin was enriched in the cell contact area and showed co-distribution with LFA-1 on the merged images (Fig. 1A). This finding was consistent after 5 minutes (not shown) and 20 minutes of incubation in $80-90 \%$ of conjugates $(n=20)$. The distribution of F-actin (red) and LFA-1 (green) was further analyzed by $3 \mathrm{D}$ reconstruction of the confocal images and the analysis of a cross-section of the cell-cell interface from CTL side. Three examples of such analysis are shown in Fig. (1B). In all 15 CTL-L cell contact sites analyzed by the $3 \mathrm{D}$ reconstruction, F-actin formed an outer-ring. LFA-1 partially co-distributed with actin and localized in the ring in $60 \%$ of CTLs examined, although LFA1 did not completely overlap with actin and was often enriched in one side of the ring on the merged images (Fig. 1B). We also examined the distribution of WASP in CTLs, which accumulated at the cell contact site (Fig. 1C, D). To quantify the distribution of LFA-1, WASP and actin, we calculated the ratio of fluorescence intensity of the site of 

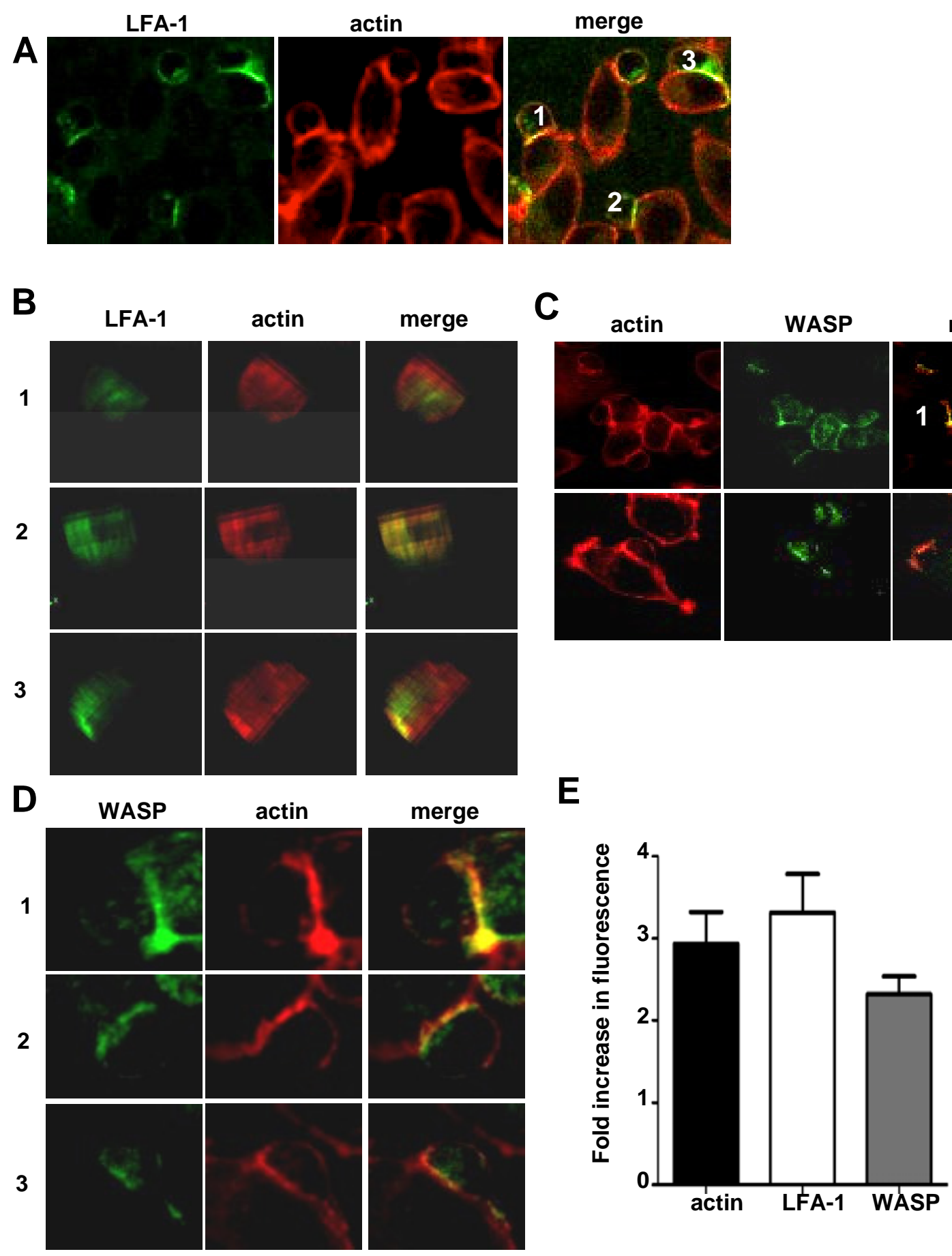

Fig. (1). LFA-1, actin and WASP accumulate in an antigen-independent immunological synapse on CTLs. CTLs were generated in vitro by stimulating HY-specific TCR transgenic mouse lymphocytes with B6 mouse dendritic cells pulsed with HY peptide and cultured in the presence of IL-2. They were incubated with ICAM-1-transfected L cells for 20 minutes, fixed, permeabilized and stained for LFA-1, actin and WASP and analyzed by confocal microscopy. (A) Three representative conjugates stained for LFA-1 and actin from more than 50 images collected are shown. Cells numbered 1-3 in the merged image (right panel) were selected for 3D reconstruction. (B) The distribution of LFA-1 and actin at CTL-L cell contact sites of the conjugates numbered in (A) were analyzed. The 3D images were reconstructed using Volocity software from the stacks of confocal images of the cell contact sites and rotated to obtain the images of the CTL-target interphase from CTL side. (C) Three representative conjugates stained for WASP and actin from more than 20 images collected are shown. Cells numbered 1-3 in the merge image (right panel) are shown at a higher magnification in (D). (E) The increase in fluorescence intensity of LFA-1, WASP and actin staining in CTLs at the site of contact with L cells was calculated. Shown is a ratio of fluorescence intensity of actin (black bar), LFA-1 (white bar) and WASP (grey bar) at the contact site in comparison with fluorescence intensity of a section of membrane of equal area located on the opposite side of the same cell (mean \pm S.E.M., $n=10-20$ ). L cells express actin but not LFA-1 or WASP. Therefore, to calculate the increase in F-actin fluorescence in CTLs at the contact site, F-actin fluorescence of L cells measured at a non-contact site of the same size was subtracted. The resulting value was used to calculate the ratio of actin accumulation as described for LFA-1 and WASP. All analyses were performed using Volocity software. 
contact between CTLs and L cells over that of a section of membrane of equal area from the opposite side of the CTL. Since L cells also express F-actin, actin staining of a noncontact site of $\mathrm{L}$ cells was subtracted from that at the cell contact site to estimate the actin intensity of CTLs at the contact site. The results showed significant enrichment of LFA-1, WASP and actin at CTL-L cell contact site (Fig. 1E). These results suggest that binding of LFA-1 on CTL to ICAM-1 on target cells results in the accumulation of actin and WASP in an antigen-independent manner.

\section{LFA-1 Capping Does Not Induce Co-Capping of F-Actin or Talin}

The above results showed accumulation of F-actin, talin and WASP in the immunological synapse of CTLs. To determine whether this is due to constitutive association of LFA-1 on CTLs with the actin cytoskeleton, a co-capping experiment was carried out. Capping of LFA-1 was induced by cross-linking with anti-LFA- $1 \mathrm{mAb}$ at $37^{\circ} \mathrm{C}$ to increase membrane fluidity and allow passive diffusion of LFA-1. The cells were fixed, permeabilized and stained for F-actin. Co-capping was assessed by confocal microscopy. We have previously shown that LFA-1 capping induced co-capping of CD3 in CTLs [4]. In control cells, in which cells were kept on ice to prevent capping, LFA-1 and actin were evenly distributed along the plasma membrane (Fig. 2A, first row). Antibody-mediated cross-linking induced striking localization of LFA-1 at one site, forming a "cap" (green), whereas F-actin remained evenly distributed (Fig. 2A, second row). This was observed in $94 \pm 5 \%$ of capped cells $(n=30)$. We also tested the distribution of talin. In control CTLs without LFA-1 capping, talin showed patchy distribution throughout the cytosol and along the plasma membrane (Fig. $\mathbf{2 A}$, row 3 ), and capping of LFA-1 did not result in co-capping of talin (Fig. 2A, bottom row). The accumulation of LFA-1, but not actin or talin, in the cap was further quantified by calculating the ratio of fluorescence intensity of the capped area of the membrane over the intensity of a section of membrane of equal area from the opposite side of the cell. Whereas LFA-1 accumulation in the cap was quite obvious, no such accumulation of actin or talin was detected in the cap as the densities of those proteins in the LFA-1 cap were the same as those in the opposite end of the cell (Fig. 2B). Thus, LFA-1 does not seem to be constitutively associated with actin or talin in CTLs.

Binding of ICAM-1-Coated Beads, But Not Anti-LFA-1 mAb-Coated Beads, Induces Accumulation of Talin, Actin and WASP at the Binding Site

Since the interaction between CTLs and L cells described above may involve multiple protein-protein interactions, we used cell-size plastic beads coated with recombinant soluble ICAM-1 or anti-LFA-1 mAb in place of ICAM-1-transfected $\mathrm{L}$ cells. The densities of these proteins on the beads, as determined by staining with appropriate antibodies and flow cytometric analysis, were comparable (data not shown). CTLs and the beads were incubated for 20 minutes at $37^{\circ} \mathrm{C}$, fixed, permeabilized, stained for talin, F-actin and WASP and examined by confocal microscopy. When CTLs bound to ICAM-1-coated beads, the shape of CTLs significantly
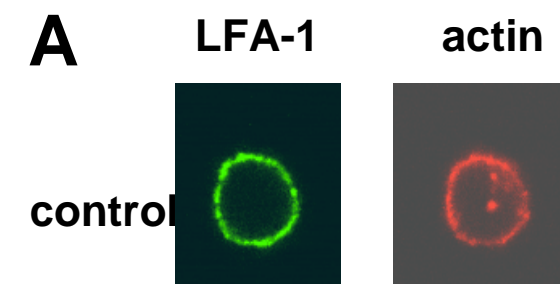

merged
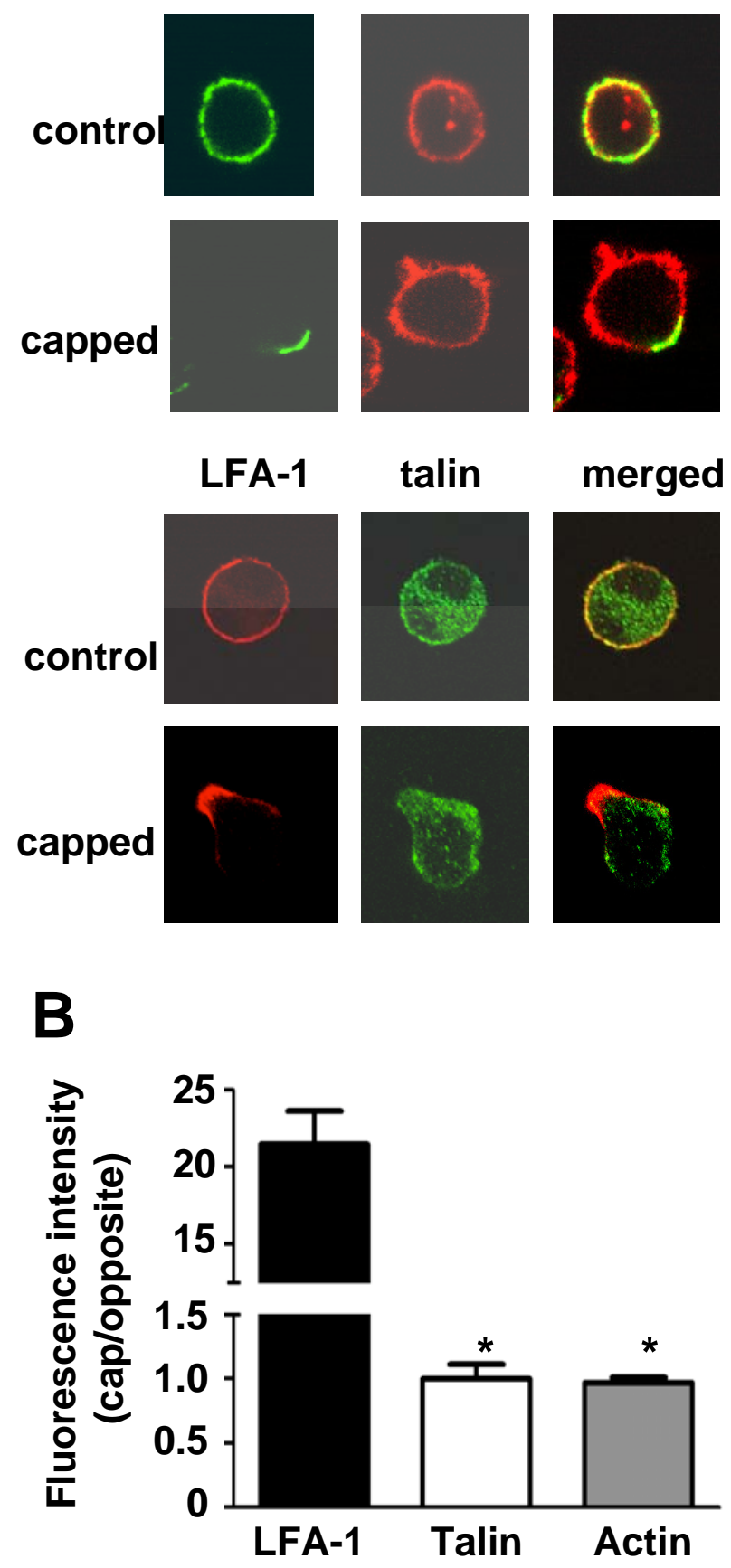

Fig. (2). Actin and talin do not co-cap with LFA-1. CTLs were incubated at $37^{\circ} \mathrm{C}$ with anti-LFA-1 $\mathrm{mAb}$ to induce capping of LFA1 , fixed, permeabilized and stained for actin or talin. The cells were analyzed by confocal microscopy. Control cells were incubated on ice to prevent capping of LFA-1, then fixed and stained with antiLFA-1. (A) Images of the mid sections of the cells are shown. Shown are representative cells from more than 30 cells for each condition. (B) Fluorescent intensity of staining shown in (A) was further analyzed to quantify the intensity of the capped area. Shown is a ratio of intensity of the capped area of the membrane over the intensity of a section of membrane of equal area from the opposite side of the cell (mean \pm S.E.M., $n=20$ ) analyzed using Volocity software. Asterisks indicate $\mathrm{p}<0.05$. 
changed. They flattened and formed lamellipodia-like membrane extensions that cupped the beads (Fig, 3A, top row). Actin, talin and WASP accumulated at the lamellipodia-like structure. In contrast, CTLs bound to anti-LFA-1 mAbcoated beads did not change their shapes and did not form lamellipodia-like structure. Furthermore, no significant enrichment of F-actin, talin or WASP at the site of beadbinding was detected (Fig. 3A, bottom row). The confocal images were further analyzed to quantify the distribution of actin in CTLs bound to ICAM-1-coated beads and anti-LFA-
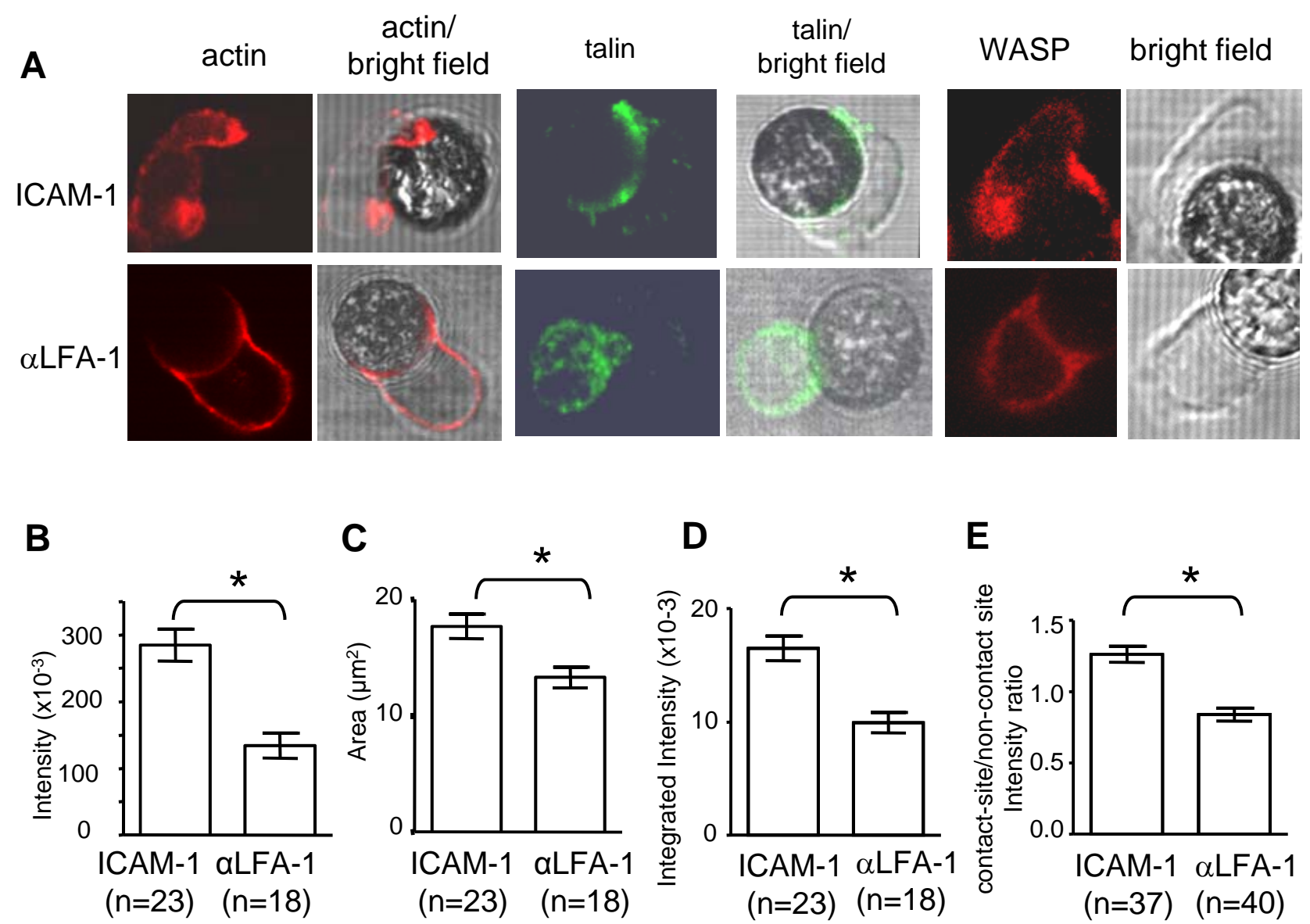

C

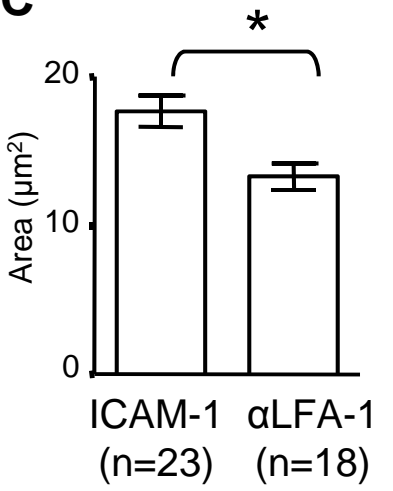

D

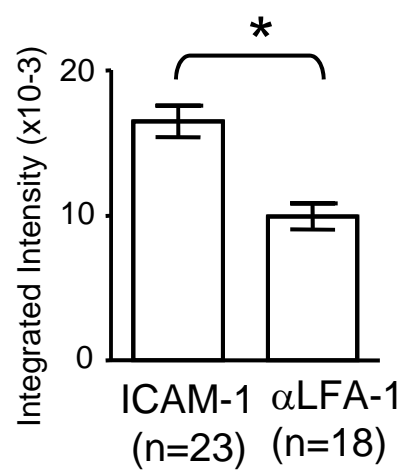

$\mathbf{E}$

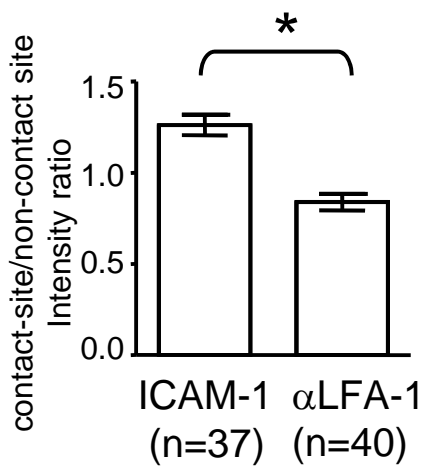

$\mathbf{F}$

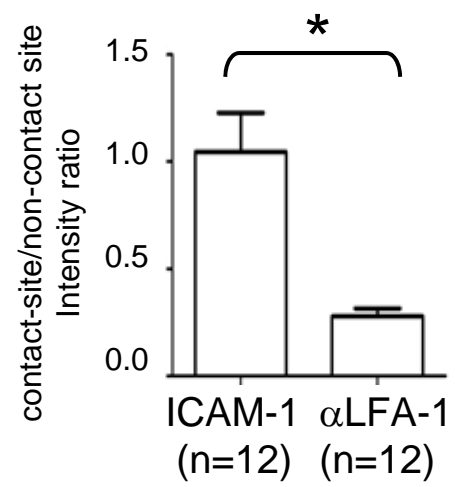

G

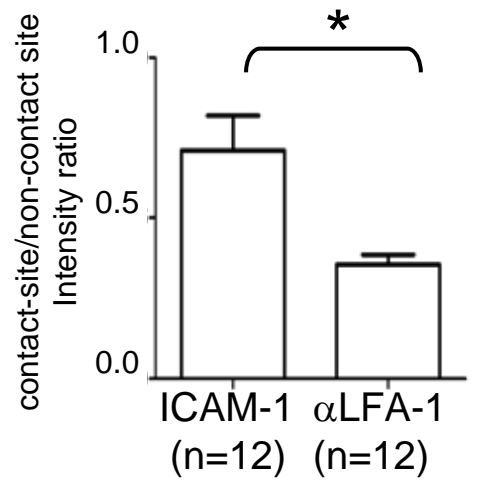

Fig. (3). Binding of ICAM-1-coated beads, but not anti-LFA-1 mAb-coated beads, induces accumulation of actin, talin and WASP at the binding site. (A) CTL were incubated with beads (10 $\mu \mathrm{m}$ diameter) pre-coated with recombinant soluble ICAM-1 or anti-LFA-1 mAb for 30 minutes at $37^{\circ} \mathrm{C}$, fixed, permeabilized and stained for actin, talin and WASP. The stained cells were analyzed by confocal microscopy. At least 50 cell-bead conjugates were analyzed for each condition. Representative images of the mid section of the cell-bead conjugates are shown. Staining analyzed in (A) was further analyzed to quantify the distribution of actin (B through E), WASP (F), and talin (G). The intensity of actin staining in the contact area $(\mathbf{B})$, the size of the contact area $(\mathbf{C})$, the integrated intensity $(\mathbf{D})$ and the ratio of the contact site intensity over non-contact site intensity (E) were determined from 18 to 40 confocal images using Volocity software (Improvision). The ratio of the contact intensity over non-contact site intensity was determined for WASP (F) and talin (G) staining from 12 images per condition using Volocity software (Improvision). Error bars represent S.E.M. Asterisks indicate $\mathrm{p}<0.05$. 
$1 \mathrm{mAb}$-coated beads. The intensity of actin staining (Fig. 3B), the bead-contact area (Fig. 3C), the integrated intensity of actin staining at the contact site (Fig. 3D) and the ratio of actin staining intensity between the bead contact site and non-contact sites of the same cells (Fig. 3E) were determined by analyzing 18 to 40 cell images by Volocity software (Improvision, Lexington, MA). By all the measurements, CTLs bound to ICAM-1-coated beads, but not anti-LFA-1 mAbcoated beads, showed significant accumulation of F-actin, WASP (Fig. 3F), and talin (Fig. 3G) at the bead-contact site. Prolonged incubation of CTLs with anti-LFA-1 mAbcoated beads up to $2 \mathrm{~h}$ did not induce redistribution of actin, talin or WASP (data not shown). These results suggest that binding of ICAM-1 to LFA-1, but not antibody-mediated LFA-1 clustering, induces stimulatory signals in CTLs leading to accumulation of F-actin, talin and WASP.

\section{Binding to ICAM-1-Coated Plates as well as Anti-LFA-1- Coated Plates Induces Pyk2 Phosphorylation}

The above results showed that binding of CTLs to antiLFA-1 mAb-coated beads does not induce accumulation of actin, talin and WASP. To test whether antibody-mediated clustering of LFA-1 resulted in any outside-in signals, we examined Pyk2 phosphorylation. Binding of LFA-1 to sICAM-1 treated plates has been shown to induce Pyk2 phosphorylation [20]. For this study, CTLs were incubated in Petri dishes coated with ICAM-1 or anti-LFA-1 mAb for various time periods, Pyk2 was immunoprecipitated and phosphorylation of tyrosine residues was examined by western blotting. Pyk 2 was rapidly phosphorylated within $5 \mathrm{~min}-$ utes in CTLs bound to an anti-LFA-1-coated dish as well as those bound to an ICAM-1-coated dish (Fig. 4A). Quantification of the ratio between phosphorylated signal and Pyk2 control showed that in both conditions the intensity of signal increased more than 3 -fold at the 5 minute time-point relative to the loading control and slowly decreased over 60 minutes (Fig. 4B). Therefore, binding of CTLs to anti-LFA-1 coated plastic surface induces stimulatory signals in CTLs leading to Pyk2 phosphorylation but not accumulation of actin, talin or WASP.

\section{Disruption of Actin Cytoskeleton by Cytochalasin D Treatment Inhibits LFA-1 Mediated Adhesion to ICAM-1}

It has been reported that LFA-1 on resting human B lymphoid line is immobile on the plasma membrane due to its association with the actin cytoskeleton and treatment with a
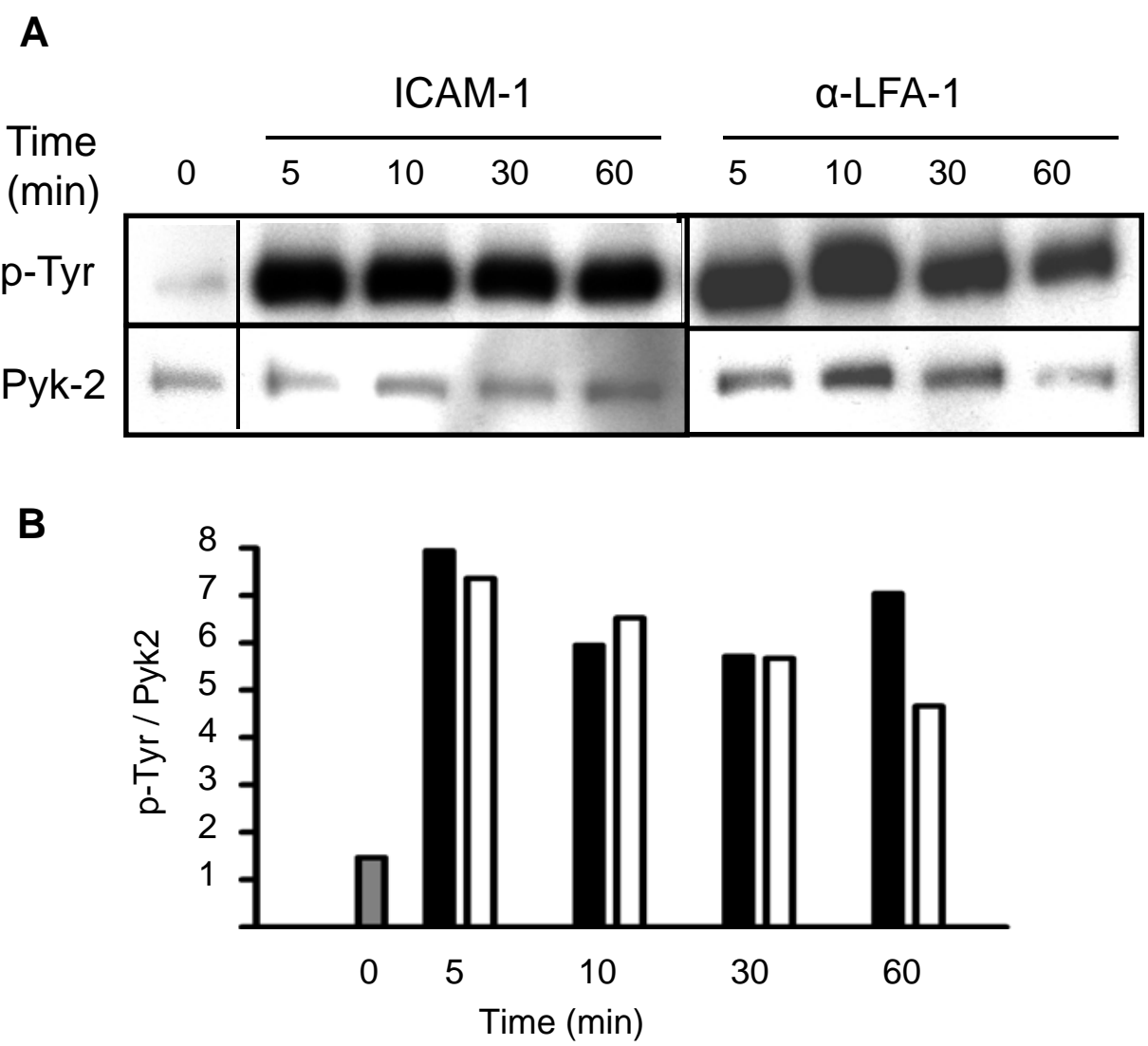

Fig. (4). Tyrosine phosphorylation of Pyk-2 is enhanced in CTLs after binding to both ICAM-1 and anti-LFA-1 mAb coated surfaces. (A) CTLs were allowed to bind to polystyrene Petri dishes coated with recombinant soluble ICAM-1 or anti-LFA-1 mAb for the indicated times (in minutes) at $37^{\circ} \mathrm{C}$ and then solubilized with ice cold lysis buffer. Time zero samples were lysed in a microfuge tube with ice cold lysis buffer. Pyk2 was immunoprecipitated as described in Materials and Methods. Immunoprecipitated samples were divided in half and analyzed for Pyk-2 (loading control) and tyrosine phosphorylation with anti-Pyk-2 and anti-phosphotyrosine (4G10) primary Abs, respectively, followed by anti-goat IgG and anti-mouse IgG secondary Abs conjugated with horseradish peroxidase. Detection was by ECL chemiluminescence. The results are representative of four independent experiments. (B) The integrated density for each band on the western blot shown in (A) was calculated using ImageJ software. Shown is the ratio of phospho-tyrosine signal to loading control signal for each time point. Grey bar shows control (0 minute), black bars show ICAM-1 and white bar show anti-LFA-1. 
low dose cytochalasin D increases the mobility of LFA-1 on the cell surface, which allows clustering of LFA-1 and enhances LFA-1-mediated cell adhesion [16]. We tested whether similar effects of cytochalasin D treatment are seen with CTLs. As LFA-1 on CTLs is in a high avidity conformation, it readily mediated cell adhesion to ICAM-1 without prior stimulation with PMA, and the adhesion was almost completely inhibited by anti-LFA-1 antibody. Surprisingly, cytochalasin D treatment, even at low doses, inhibited cell adhesion (Fig. 5A). As a control, we also examined primary splenic T cells. Adhesion of splenic T cells to immobilized ICAM-1 requires activation with PMA. The cell adhesion to ICAM-1 was effectively inhibited by anti-LFA-1 antibody. Treatment with cytochalasin D at low concentrations did not enhance LFA-1-mediated adhesion of primary splenic $\mathrm{T}$ cells treated with PMA, and higher doses inhibited adhesion, although not to the extent seen in CTLs (Fig. 5B). Adhesion of primary cells not treated with PMA was minimal, and cytochalasin D treatment produced no significant effect on adhesion of these cells. Finally, we tested the effect of cytochalasin D treatment on the EL4 murine lymphoma cell line. Like primary T cells, PMA stimulation is required for adhesion of EL4 to ICAM-1. Unlike primary cells and CTLs, EL4 cells showed increased adhesion following a low dose of cytochalasin D, although at higher concentrations adhesion was inhibited (Fig. 5C). Microscopic observation of CTLs bound to immobilized ICAM-1 showed that they flatten and often formed lamellipodia-like extensions whereas cytochalasin treatment inhibited cell flattening and formation of membrane extensions (Fig. 5D). The short incubation with cytochalasin had no effect on the viability of the cells in any of the experiments.

\section{DISCUSSION}

The reorganization of the actin cytoskeleton in effector cells is the essential first step in cell-mediated cytotoxicity. Upon binding to target cells, CTLs polarize and F-actin accumulates at the immunological synapse. Cytotoxic granules translocate and are secreted through the synapse [21]. We have previously shown that binding of CTLs to cells expressing ICAM-1 leads to antigen-independent formation of an immunological synapse in which LFA-1 and CD3 are codistributed in a ring. The actin cytoskeleton also accumulates in the antigen-independent immunological synapse of CTLs [4]. We now have shown that the reorganization of the actin cytoskeleton in CTLs requires binding of ICAM-1 to LFA-1 on CTLs, independent of TCR signalling. When CTLs bind to cells expressing ICAM-1 or plastic beads coated with recombinant ICAM-1, F-actin and WASP accumulate at the binding site. This is not due to simple clustering of LFA-1, or constitutive association of F-actin and WASP with LFA1 , as binding of anti-LFA-1 mAb-coated beads does not induce the same redistribution of these proteins. Antibodymediated capping of LFA-1 also fails to induce co-capping of F-actin or talin. Without binding of ICAM-1 to LFA-1, cortical actin in CTLs is evenly distributed underneath the plasma membrane whereas talin and WASP are distributed throughout the cytosol. Thus, ligand binding to LFA-1 transduces outside-in signals that lead to the reorganization of the actin cytoskeleton. The precise signaling pathways involved in this are still unclear.
Binding of talin to the cytoplasmic tail of integrins, including LFA-1, is thought to be the final step of integrin activation [6]. Because LFA-1 on CTLs used in this study is pre-activated and readily mediates CTL adhesion to ICAM1, talin in CTLs may be expected to constitutively associate with LFA-1. However, we see no sign of physical association of talin with LFA-1 in CTLs unless ICAM-1 binds to LFA-1. In addition to the confocal microscopy study presented above, we have attempted to co-immunoprecipitate talin with LFA-1 without success (data not shown). The head domain of talin, which has high affinity for the cytoplasmic tail of LFA-1, is thought to be generated upon cell activation by proteolytic cleavage of talin with the calcium-dependent proteases calpains [22]. However, western blotting with talin head-specific $\mathrm{mAb}$ detects only full length talin, but not free talin head, in CTLs in suspension or bound to immobilized ICAM-1 (data not shown). Therefore, the association between talin and LFA-1 seems to be induced by ligand binding to LFA-1 rather than calpain-mediated cleavage of talin. Kim et al. have shown that ligand binding induces separation of the cytoplasmic domains of the two chains of LFA-1 [8]. It is conceivable that this exposes the talin-binding site on the cytoplasmic tail of LFA-1 and recruits talin.

The mechanisms by which ICAM-1 binding to LFA-1 induces accumulation of F-actin and WASP in the binding site are still unknown. Since talin has multiple actin-binding domains, it is possible that F-actin passively accumulates by its association with talin. On the other hand, accumulation of WASP suggests that ligand binding of LFA-1 may transduce activation signals to recruit and activate WASP, leading to Arp2/3-mediated actin polymerization. WASP has been shown to be recruited to the immunological synapse formed in T cells as they interact with APC [23]. However, the activation and recruitment of WASP to the immunological synapse is thought to be mediated by activation signals transduced by the TCR, involving activation of Cdc42, ZAP-70 and Protein Kinase $\theta$ [24]. In CTLs in this study, the accumulation of WASP in the immunological synapse is independent of antigen-TCR interaction and is induced by ICAM-1 binding to LFA-1. Sanchez-Madrid et al. reported that LFA-1 ligation induces Vav1-mediated Rac-1 activation [24]. Since Vav1-mediated Rac activation is thought to be an important step for actin polymerization [25], we have examined the effects of ICAM-1 binding to LFA-1 on Vav1, but it seems to be constitutively phosphorylated in CTLs used in this study, and ICAM-1 binding has no significant effect on Vav1 activation (data not shown). Binding of ICAM-2 to the human $\mathrm{T}$ cell line Jurkat induces phosphorylation of the $\beta$ chain and release of Jun-activating binding protein (JAB-1) and phosphorylation of cytohesin-1, which leads to Erk1/2 phosphorylation [26]. In neutrophils, integrin-mediated signaling requires the Syk tyrosine kinase [27], and $\beta 2$ integrinmediated activation is defective in double mutant neutrophils and macrophages deficient for both DAP12 and FcR $\gamma$ [28]. Whether the same signaling pathway is involved in LFA-1mediated activation in CTLs is unknown.

An interesting finding in this study is the difference between binding of ICAM-1 and anti-LFA- 1 mAb. As discussed above, ICAM-1 binding likely induces conformational changes in LFA-1, which is not achieved by antibodymediated cross-linking or clustering of LFA-1. Antibody- 
A

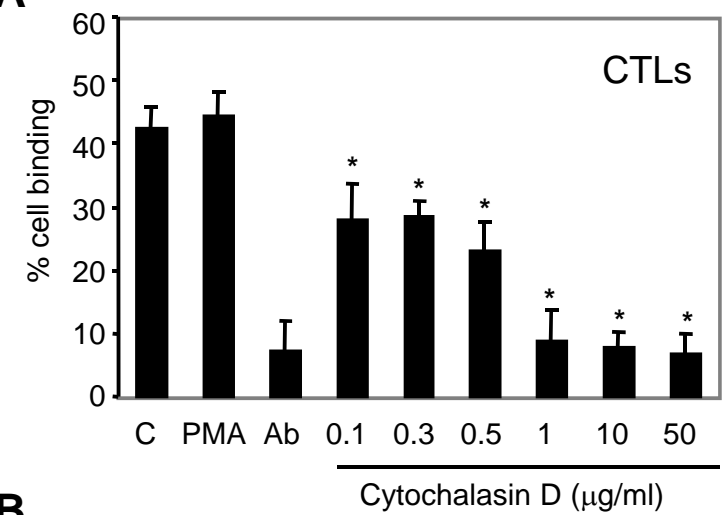

B

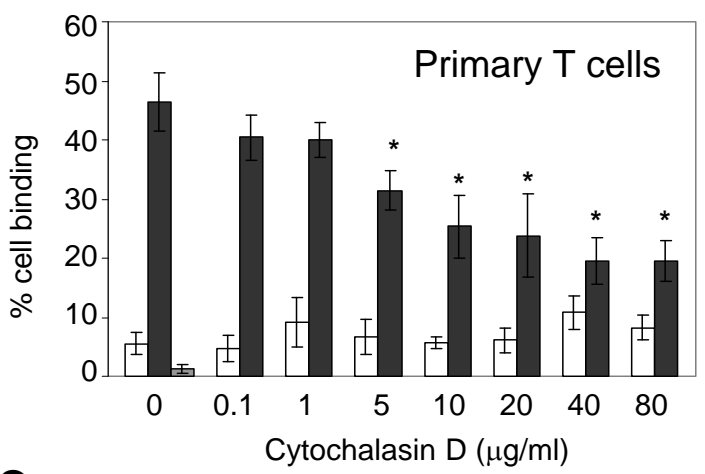

C

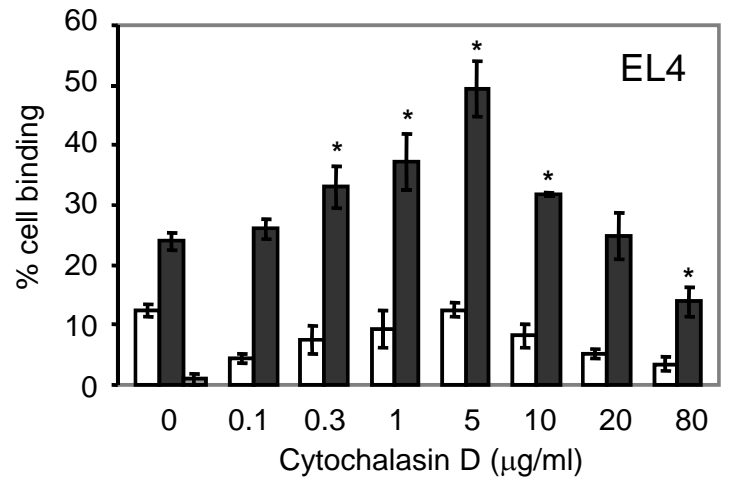

D

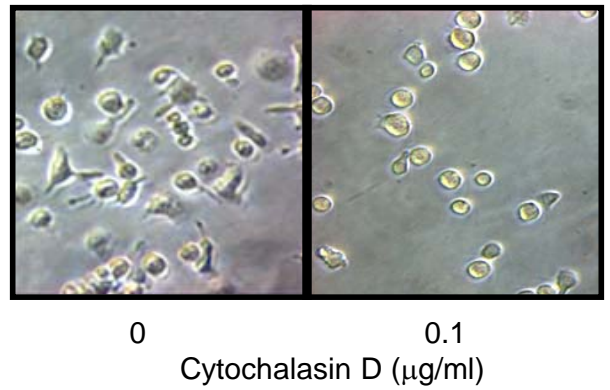

Fig. (5). Cytochalasin treatment inhibits adhesion of CTLs and primary T cells, but enhances adhesion of the EL4 cell line. CTL (A), primary splenic T cells (B), or EL4 cells (C) were labeled with Calcein-AM, washed, and treated with 50 ng/ml PMA (indicated by PMA label in (A) and black bars in $(\mathbf{B})$ and $(\mathbf{C})$ ) and the indicated amount of cytochalasin D (C label in (A) denotes no treatment with cytochalasin D or PMA). Some cells were treated with anti-LFA-1 blocking mAb to confirm specificity of binding to ICAM-1 (indicated by Ab label in (A) and grey bars in (B) and (C)). Cells were allowed to bind to plates which had been coated with soluble recombinant ICAM-1, then fluorescence was read, plates were washed to remove non-adherent cells, and fluorescence as read again. Cell adhesion was calculated as a ratio of pre- to post- wash values. Results shown are representative of at least 3 independent experiments, each done in triplicate. Error bars indicate S.E.M. Asterisks denote p < 0.05 compared to PMA-stimulated control (indicated by PMA label in (A) and black bars in (B) and (C)). (D) CTLs treated with indicated concentration of cytochalasin D were subjected to the cell adhesion assay above. After washing away nonadherent cells, cells that remained adhered to the wells were examined with an inverted phase contrast microscope and photographed. 
mediated cross-linking is often used to study LFA-1 signaling. Our current study has shown that antibody-mediated clustering of LFA-1 does induce some signaling such as Pyk2 phosphorylation. Similarly, Suzuki et al. have recently reported that antibody-mediated cross-linking of LFA-1 on T cells results in a ring-shaped organization of actin, termed "actin cloud", which involves adhesion- and degranulationpromoting adaptor protein (ADAP) phosphorylation and cJun N-terminal kinase (JNK) activation [29]. Our study has clearly shown that binding of immobilized ICAM-1 and immobilized anti-LFA-1 mAb have strikingly different effects on the distribution of actin, talin and WASP and indicated that ligand binding is critical for the reorganization of the actin cytoskeleton in CTLs.

Finally, we have examined the effect of actin inhibition by cytochalasin D on CTL. Previous reports have suggested that cytochalasin D treatment at low doses may enhance cell adhesion by releasing LFA-1 from the actin cytoskeleton, thus making it more mobile on the cell surface. We were surprised to find that even low doses of cytochalasin D resulted in inhibition of LFA-1 mediated adhesion to ICAM-1. As a control, we also tested primary T cells. As these splenic $\mathrm{T}$ cells were resting, activation by PMA was required for their adhesion to ICAM-1. PMA-induced adhesion of primary $\mathrm{T}$ cells was also inhibited by cytochalasin $\mathrm{D}$ treatment. In contrast, treatment of the EL4 murine T cell lymphoma cell line with a low dose of cytochalasin $\mathrm{D}$ resulted in an increase in adhesion, while a high dose impaired adhesion. This is consistent with previous findings using EBV transformed B cells [16]. It is unclear why low dose cytochalasin $\mathrm{D}$ inhibits adhesion of CTLs and primary $\mathrm{T}$ cells while it enhances adhesion of cell lines. It may be due to differences in the interaction between LFA-1 and the actin cytoskeleton. It should also be noted that cytochalasin D treatment, even at high concentrations, had no effect on antibody-mediated LFA- 1 capping on CTLs or the distribution of LFA- 1 on the cell surface (data not shown). This suggests that, in CTLs, cytochalasin does not release LFA-1 from constitutive association with actin, and instead may be impairing actin accumulation required for firm cellular adhesion. Visualization by microscopy of cytochalasin D treated CTLs bound to an ICAM-1 coated surface shows a distinct difference in cellular morphology, with untreated cells appearing flat and spread, and treated cells appearing rounder, with fewer cellular projections. This difference in appearance also suggests that actin may be required for cells to firmly adhere to ICAM-1. These results, combined with the confocal microscopy results, suggest that the actin accumulation that occurs following binding of LFA-1 to ICAM-1, but not anti-LFA-1 cross-linking, is required for adhesion of T cells to ICAM-1 coated surfaces. We are currently investigating whether recruitment of actin following binding to ICAM-1 is talin- and WASP- dependent, and are thus working to elucidate an important pathway in immune synapse formation that appears to be dependent upon outside-in integrin signaling.

\section{ACKNOWLEDGEMENT}

This work was supported by a grant from the Canadian Institutes of Health Research.

\section{REFERENCES}

[1] Springer TA, Davignon D, Ho MK, Kurzinger K, Martz E, Sanchez-Madrid F. LFA-1 and lyt-2,3, molecules associated with T lymphocyte-mediated killing; and mac-1, an LFA-1 homologue associated with complement receptor function. Immunol Rev 1982; 68: 171-95.

[2] Dustin ML, Bivona TG, Philips MR. Membranes as messengers in T cell adhesion signaling. Nat Immunol 2004; 5: 363-72.

[3] Porter JC, Bracke M, Smith A, Davies D, Hogg N. Signaling through integrin LFA-1 leads to filamentous actin polymerization and remodeling, resulting in enhanced $\mathrm{T}$ cell adhesion. J Immunol 2002; 168: 6330-5.

[4] Marwali MR, MacLeod MA, Muzia DN, Takei F. Lipid rafts mediate association of LFA-1 and CD3 and formation of the immunological synapse of CTL. J Immunol 2004; 173: 2960-7.

[5] Somersalo K, Anikeeva N, Sims TN, et al. Cytotoxic T lymphocytes form an antigen-independent ring junction. J Clin Invest 2004; 113: 49-57.

[6] Tadokoro S, Shattil SJ, Eto K, et al. Calderwood, Talin binding to integrin beta tails: A final common step in integrin activation. Science 2003; 302: 103-6.

[7] Calderwood DA, Zent R, Grant R, Rees DJ, Hynes RO, Ginsberg MH. The talin head domain binds to integrin beta subunit cytoplasmic tails and regulates integrin activation. J Biol Chem 1999; 274: 28071-4.

[8] Kim M, Carman CV, Springer TA. Bidirectional transmembrane signaling by cytoplasmic domain separation in integrins. Science 2003; 301: 1720-5.

[9] Calderwood DA, Yan B, de Pereda JM, et al. The phosphotyrosine binding-like domain of talin activates integrins. J Biol Chem 2002; 277: 21749-58.

[10] Vinogradova O, Velyvis A, Velyviene A, et al. A structural mechanism of integrin alpha(IIb)beta(3) "inside-out" activation as regulated by its cytoplasmic face. Cell 2002; 110: 587-97.

[11] Vinogradova O, Vaynberg J, Kong X, Haas TA, Plow EF, Qin J. Membrane-mediated structural transitions at the cytoplasmic face during integrin activation. Proc Natl Acad Sci USA 2004; 101: 4094-9.

[12] Badour K, Zhang J, Siminovitch KA. The wiskott-aldrich syndrome protein: Forging the link between actin and cell activation. Immunol Rev 2003; 192: 98-112.

[13] Badour K, Zhang J, Shi F, et al. The wiskott-aldrich syndrome protein acts downstream of CD2 and the CD2AP and PSTPIP1 adaptors to promote formation of the immunological synapse. Immunity $2003 ; 18: 141-54$.

[14] Cannon JL, Burkhardt JK. Differential roles for wiskott-aldrich syndrome protein in immune synapse formation and IL-2 production. J Immunol 2004; 173: 1658-62.

[15] Orange JS, Ramesh N, Remold-O'Donnell E, et al. Wiskott-aldrich syndrome protein is required for NK cell cytotoxicity and colocalizes with actin to NK cell-activating immunologic synapses. Proc Natl Acad Sci USA 2002; 99: 11351-6.

[16] Kucik DF, Dustin ML, Miller JM, Brown EJ. Adhesion-activating phorbol ester increases the mobility of leukocyte integrin LFA-1 in cultured lymphocytes. J Clin Invest 1996; 97: 2139-44.

[17] Takei F. Inhibition of mixed lymphocyte response by a rat monoclonal antibody to a novel murine lymphocyte activation antigen (MALA-2). J Immunol 1985; 134: 1403-7.

[18] Welder CA, Lee DH, Takei F. Inhibition of cell adhesion by microspheres coated with recombinant soluble intercellular adhesion molecule-1. J Immunol 1993; 150: 2203-10.

[19] Marwali MR, Rey-Ladino J, Dreolini L, Shaw D, Takei F. Membrane cholesterol regulates LFA-1 function and lipid raft heterogeneity. Blood 2003; 102: 215-22.

[20] Rodriguez-Fernandez JL, Gomez M, Luque A, Hogg N, SanchezMadrid F, Cabanas C. The interaction of activated integrin lymphocyte function-associated antigen 1 with ligand intercellular adhesion molecule 1 induces activation and redistribution of focal adhesion kinase and proline-rich tyrosine kinase 2 in T lymphocytes. Mol Biol Cell 1999; 10: 1891-907.

[21] Stinchcombe JC, Bossi G, Booth S, Griffiths GM. The immunological synapse of CTL contains a secretory domain and membrane bridges. Immunity 2001; 15: 751-61. 
[22] Stewart MP, McDowall A, Hogg N. LFA-1-mediated adhesion is regulated by cytoskeletal restraint and by a $\mathrm{Ca} 2+-$ dependent protease, calpain. J Cell Biol 1998; 140: 699-707.

[23] Sasahara Y, Rachid R, Byrne MJ, et al. Mechanism of recruitment of WASP to the immunological synapse and of its activation following TCR ligation. Mol Cell 2002; 10: 1269-81.

[24] Sanchez-Martin L, Sanchez-Sanchez N, Gutierrez-Lopez MD, et al. Signaling through the leukocyte integrin LFA-1 in T cells induces a transient activation of rac-1 that is regulated by vav and PI3K/Akt-1. J Biol Chem 2004; 279: 16194-205.

[25] Miletic AV, Swat M, Fujikawa K, Swat W. Cytoskeletal remodeling in lymphocyte activation. Curr Opin Immunol 2003; 15: 261-8.

[26] Perez OD, Mitchell D, Jager GC, et al. Leukocyte functional antigen 1 lowers $\mathrm{T}$ cell activation thresholds and signaling through cytohesin-1 and jun-activating binding protein 1. Nat Immunol 2003; 4: 1083-92.

[27] Mocsai A, Zhou M, Meng F, Tybulewicz VL, Lowell CA. Syk is required for integrin signaling in neutrophils. Immunity 2002; 16: 547-58.

[28] Mocsai A, Abram CL, Jakus Z, Hu Y, Lanier LL, Lowell CA. Integrin signaling in neutrophils and macrophages uses adaptors containing immunoreceptor tyrosine-based activation motifs. Nat Immunol 2006; 7: 1326-33.

[29] Suzuki J, Yamasaki S, Wu J, Koretzky GA, Saito T. The actin cloud induced by LFA-1-mediated outside-in signals lowers the threshold for T-cell activation. Blood 2007; 109: 168-75.

(C) Mace et al.; Licensee Bentham Open .

This is an open access article licensed under the terms of the Creative Commons Attribution Non-Commercial License (http://creativecommons.org/licenses/by$\mathrm{nc} / 3.0 /$ ) which permits unrestricted, non-commercial use, distribution and reproduction in any medium, provided the work is properly cited. 